\title{
Regulating a Monopolist With UnKnown Demand: Costly Public Funds and the Value OF PRIVATE INFORMATION
}

\author{
IÑAKI AGUIRRE and ARANTZA BEITIA \\ Universidad del País Vasco
}

\begin{abstract}
In this paper, we analyze the optimal regulation policy when the regulated firm has better information concerning the market demand than the regulator. We show that introducing a cost on public funds into the Planner's objective function does not lead to qualitative results similar to those obtained by introducing distributional considerations. In particular we show that under constant marginal cost the full information policy is not implementable and that the optimal regulatory policy results in informational rents. The social value of private information and the firm's informational rents are both increasing functions of the cost of the public funds.
\end{abstract}

\section{Introduction}

The theoretical literature on regulation typically assumes that the regulated firm has more information about its technology than the regulator. The firm is usually assumed to be an informational monopoly that has private information concerning some cost parameters. The price of the product is established by the regulator who is allowed to operate monetary transfers to the regulated firm. The application of the principal-agent method leads to well known results: asymmetry of information causes a loss of efficiency due to the necessity of limiting monopoly informational rents. As a consequence, a higher price is charged to consumers and the quantity of the product is therefore reduced.

Iñaki Aguirre and Arantza Beitia, Departamento de Fundamentos del Análisis Económico I, Universidad del País Vasco, Avenida Lehendakari Aguirre 83, 48015 Bilbao, Spain ( jepagpei@bs.ehu.es) ( jepberua@bs.ehu.es).

We are grateful to two anonymous referees, and to participants in the 28th Annual Conference of the European Association for Research in Industrial Economics, EARIE, (Dublin) and the I Encuentro de Economía Industrial (Barcelona) for helpful comments. Financial support from BEC (2000-0301) and UPV (035.321-HB8238/2000) is gratefully acknowledged.

Received January 2002; Accepted May 2003.

(c) 2004 Blackwell Publishing, Inc.

Journal of Public Economic Theory, 6 (5), 2004, pp. 693-706. 
These results depend crucially on the deviation of the regulator's objective function from the standard first best analysis where the regulator maximizes the sum of the surplus of consumers and producers. In the literature on government regulation these deviations are produced in two ways. Either distributional considerations are introduced through a coefficient $(\alpha<1)$ affecting firms' profits or costly public funds are considered: if the government transfers $\$ 1$ to the firm, society pays $\$(1+\lambda)$. ${ }^{1}$ The two formulations lead to similar qualitative results under asymmetric information on costs.

Although the analysis of the design of regulatory policies when the regulated firm is better informed concerning demand than the regulator seems to be an important issue, it has been neglected in literature. Notable exceptions are the papers by Riordan (1984) and Lewis and Sappington (1988a). ${ }^{2}$

In this paper, we analyze the optimal regulation policy when the regulated firm has better information concerning the market demand than the regulator. Lewis and Sappington (1988a) analyze the problem when the objective function of the regulator introduces distributional considerations. They demonstrate that when a monopolist's marginal costs are nondecreasing, its private information about demand is inconsequential for the design of optimal regulatory mechanisms. That is, the regulator will implement the same allocations as if the monopolist's knowledge about demand were public information. Thus, none of the incentive constraints is binding; the monopolist earns no information rents and will supply socially efficient levels of output. Laffont and Tirole (1993) suggest that the result of Lewis and Sappington (1988a) might not hold with a shadow price of public funds. In this paper, we demonstrate that in fact the results of Lewis and Sappington are not maintained. Moreover, we characterize the optimal regulatory policy and the contract between the regulator and the firm when the presence of costly public funds is considered. Our results, therefore, highlight some interesting differences between the two alternative formulations of the social welfare function (with costly public funds or with distributional considerations) that arise under private information on market demand.

This paper is organized as follows. Section 2 develops the basic model. Section 3 presents the full information case as a benchmark. In Section 4, we analyze the design of the optimal regulatory policy under private information on market demand. Section 5 generalizes the social welfare function to incorporate both distributional considerations and costly public funds. This setting allows us to draw a comparison between our findings and the results of Lewis and Sappington (1988a). Finally, Section 6 offers concluding remarks.

\footnotetext{
${ }^{1}$ In the public finance theory, the parameter $\lambda$ is usually called the shadow cost of public funds (see, e.g., Atkinson and Stiglitz 1980).

${ }^{2}$ Lewis and Sappington (1988b) and Armstrong (1999) consider the optimal regulation of a firm that has private information about both its cost and demand functions. On the other hand, Biglaiser and Ma (1995) consider the effects of strategic rivals on the regulatory policy under private information on consumer demand.
} 


\section{The Model}

We consider the following regulatory environment. The cost of producing output $q$ is known to be given by the function $C(q)=\mathrm{c} q+F$, where $c$ is the constant marginal cost and $F$ the fixed cost. The market demand for the product of the firm is given by $q=D(p, \theta)$, where $p$ is the unit price and the parameter $\theta$ captures the firm's private information concerning market demand. We assume that $D_{\theta}(p, \theta)>0$ and $D_{p}(p, \theta)<0$ (where subscripts denote partial derivatives), that is, the higher the realization of $\theta$ the larger the quantity of output consumers demand at any nonnegative price, and the more the output demanded the lower the regulated price. Moreover we assume that $D_{p \theta}=0 .^{3}$ The regulator's uncertainty about $\theta$ is represented by a probability distribution $F(\theta)$ with associated density function $f(\theta)$ strictly positive on the support $[\underline{\theta}, \bar{\theta}]$. The function $f(\theta)$ is common knowledge but the realization of $\theta$ is observed only by the firm.

The regulator is endowed with the power to establish a unit price $p$ for the firm's output and to specify a transfer $t$ from consumers to the firm. To simplify the analysis, we assume that consumer demand depends only on price $p .{ }^{4}$ The regulator can observe and therefore enforce the regulated price. The quantity sold at the regulated price is assumed to be too costly for the regulator to monitor directly. ${ }^{5}$ However, the regulator can still be sure that the firm fulfils its mandate to serve all demand at the regulated price. The regulator need only invite consumers to report any incident in which they were (either) refused service at the established price (or charged a higher price) and penalize the firm for such rationing.

There is a cost of public funds: we assume that raising and transferring $\$ 1$ through public channels costs society $\$(1+\lambda) .{ }^{6}$ The social welfare, the

\footnotetext{
${ }^{3}$ Any setting in which higher realizations of $\theta$ correspond to parallel outward shifts in demand constitutes a setting where $D_{p \theta}=0$. For example, $q=D(p, \theta)=g(\theta)+d(p)$, with $g^{\prime}(\theta)>0$.

${ }^{4}$ The total fixed charge may be thought of as apportioned among consumers in such a manner that no consumer is excluded from purchasing the good. The analysis can thus be conducted in terms of the aggregate fixed charges $t$ paid to the firm by consumers.

${ }^{5}$ As noticed by Lewis and Sappington (1988a), with the cost function known, if output were observable the firm might simply be compensated for its realized costs. Under this policy, the firm would have no incentive to produce a level of output other than the one most preferred by the regulator. Therefore, the problem is only interesting if the firm's sales cannot be observed perfectly by the regulator. Following Lewis and Sappington (1988a) we assume that quantities are completely unobservable to the regulator.

${ }^{6}$ Transfers between a firm and either consumers or the state may involve administrative costs, tax distortions, or inefficiencies that must be taken into account in the design of the regulatory mechanism (See, e.g., Laffont and Tirole 1986, 1993 and Caillaud et al. 1988). In other words, ideal lump-sum taxes (which would imply that $\lambda=0$ ) are not available. The measurement of the shadow cost of public funds results from the theory of public finance. A reasonable mean estimate for the U.S. economy seems to be $\lambda=0.3$ (see Ballard, Shoven, and Whalley 1985).
} 
net surplus of consumers and the profit of the firm are given respectively by

$$
\begin{aligned}
S W(p, t) & =C S(p, t)+\Pi(p, t) \\
C S(p, t) & =\int_{p}^{\infty} D(x, \theta) d x-(1+\lambda) t \\
\Pi(p, t) & =(p-c) D(p, \theta)-F+t .
\end{aligned}
$$

Therefore, social welfare can be expressed as

$$
S W(p, t)=\int_{p}^{\infty} D(x, \theta) d x+(p-c) D(p, \theta)-F-\lambda t .
$$

\section{The Full Information Case: A Benchmark}

Consider the benchmark case in which the regulator knows all components of the market demand function. The problem of the regulator under full information is given by

$$
\begin{aligned}
& \max _{p, t} S W(p, t)=\int_{p}^{\infty} D(x, \theta) d x+(p-c) D(p, \theta)-F-\lambda t \\
& \text { s.t. } \Pi(p, t) \geq 0 .
\end{aligned}
$$

Solving (3) for $t$ and substituting $t$ in (5), the problem is equivalent to

$$
\begin{aligned}
\max _{p, \Pi} S W(p, \Pi)= & \int_{p}^{\infty} D(x, \theta) d x+(1+\lambda)[(p-c) D(p, \theta)] \\
& -(1+\lambda) F-\lambda \Pi \\
& \text { s.t. } \quad \Pi \geq 0 .
\end{aligned}
$$

The solution to this problem is given by

$$
\begin{aligned}
S W_{\Pi}\left(p^{*}, \Pi^{*}\right)= & -\lambda \\
S W_{p}\left(p^{*}, \Pi^{*}\right)= & -D\left(p^{*}, \theta\right)+\left\{D\left(p^{*}, \theta\right)+\left(p^{*}-c\right) D_{p}\left(p^{*}, \theta\right)\right\} \\
& +\lambda\left\{D\left(p^{*}, \theta\right)+\left(p^{*}-c\right) D_{p}\left(p^{*}, \theta\right)\right\} \\
= & 0 .
\end{aligned}
$$

We can interpret condition (8) as follows. A marginal increase in prices reduces consumer surplus in $D(\cdot)$, the first term in (8), and increases the firm's profit in $D(\cdot)+\left(p^{*}-c\right) D p(\cdot)$, the second term. Given that the firm's profits are zero at the optimum, the regulator can reduce the transfer by the equivalent of that amount that implies, under costly public funds, a saving in social cost of $\lambda\left\{D(\cdot)+\left(p^{*}-c\right) D p(\cdot)\right\}$, the third term in (8). At the optimal 
price, the marginal social cost of a change in price equals the marginal social benefit.

We can rewrite these conditions as

$$
\begin{aligned}
\Pi^{*} & =0 \\
\frac{p^{*}-c}{p^{*}} & =\frac{\lambda}{1+\lambda} \frac{1}{\varepsilon\left(p^{*}, \theta\right)} .
\end{aligned}
$$

We obtain that the optimal price is given by the Ramsey formula: the Lerner index (or price-marginal cost ratio) is inversely proportional to the elasticity of demand. In particular, the Lerner index is $\lambda /(1+\lambda)$ times the inverse of the elasticity of demand. When $\lambda$ is zero taxation is not distortive and the optimal price is therefore equal to marginal cost. When the shadow cost of public funds becomes very large, the price tends to the monopoly price.

The full information policy consists of prices $p^{*}(\theta, \lambda)$, given by $(10)$, and transfer payments $t^{*}(\theta, \lambda)$ such that firms obtain zero profits. It is easy to check that the optimal price is an increasing function of both the consumer demand, $\theta$, and the cost of public funds, $\lambda$.

\section{Regulation under Private Information on Demand}

We now study the regulation model when the firm has private information concerning market demand. Consider that $\theta$ is continuously distributed on the support $\Theta=[\underline{\theta}, \bar{\theta}]$ according to the cumulative distribution function $F(\theta)$ and strictly positive density $f(\theta)$. We assume that $F(\theta)$ satisfies the monotone hazard rate condition; that is, the ratio $f(\theta) /[1-F(\theta)]$ is a nondecreasing function of $\theta .^{7}$

The single-crossing property, which states that the greater the demand, the more systematically willing a firm is to forego transfer payments to obtain a higher unit price, holds if the firm's marginal rate of substitution (MRS) of price for transfer payment grows with $\theta$. Given that the firm's profit is $\Pi(p, t, \theta)=p D(p, \theta)-c(D(p, \theta))-F+t$, then $\mathrm{MRS}_{p, t}=-\left(\Pi_{p} / \Pi_{t}\right)=$ $-\Pi_{p}$. As we have assumed $D_{p \theta}=0$ then $d\left|\mathrm{MRS}_{p, t}\right| / d \theta=\Pi_{p \theta} \geq 0$.

To characterize the equilibrium under private information we adopt the approach of Baron and Myerson (1982), Guesnerie and Laffont (1984), and Baron (1989). We first determine the class of feasible policies and then select the optimal policy from that class.

At the first stage, we restrict the analysis to direct revelation mechanisms by the revelation principle. ${ }^{8}$ A direct revelation mechanism is composed of

\footnotetext{
${ }^{7}$ This property requires the density function not to increase too rapidly and is satisfied by frequently used distribution functions (e.g., uniform, normal, and exponential).

${ }^{8}$ The revelation principle is established in Myerson (1979) and Dasgupta, Hammond, and Maskin (1979).
} 
transfer functions and associated price levels $\{p(\theta), t(\theta)\}_{\theta \in[\theta, \bar{\theta}]}$. Therefore, we may be restricted to regulatory policies that require the firm to report its private information parameter truthfully, that is, incentive compatible regulatory policies, to determine the class of feasible policies.

The regulator maximizes the expected social welfare subject to the incentive compatibility and individual rationality constraints. These restrictions are given as follows:

1. Incentive compatibility constraints $(I C)$ : The firm reports $\theta$ truthfully if the profit it expects to obtain by announcing its type is at least as great as the expected profit from any other report. That is,

$$
\Pi(\theta) \geq \Pi(\hat{\theta}, \theta) \quad \forall(\hat{\theta}, \theta) \in \Theta^{2},
$$

where $\Pi(\hat{\theta}, \theta)=[p(\hat{\theta})-c] D(p(\hat{\theta}), \theta)-F+t(\hat{\theta})$ and $\Pi(\theta)=\Pi(\theta, \theta)$.

2. Individual rationality constraints (IR): The regulator cannot force the firm to participate if it expects negative profits. That is,

$$
\Pi(\theta) \geq 0 \quad \forall \theta \in \Theta .
$$

The regulator's problem (RP) can be written as

$$
\begin{array}{r}
\max _{p(\theta), \Pi(\theta)} \int_{\theta \in[\theta, \bar{\theta}]}\left\{\int_{p}^{\infty} D(x, \theta) d x+(1+\lambda)[p(\theta) D(p(\theta), \theta)\right. \\
-c D(p(\theta), \theta)-F]-\lambda \Pi(p(\theta), \theta)\} f(\theta) d \theta
\end{array}
$$

subject to (IC) and (IR).

The following lemma characterizes the class of policies that satisfies (IC).

LEMMA 1: Necessary and sufficient conditions for (IC) are

(i) $\frac{d \Pi(\theta)}{d \theta}=\Pi_{\theta}=[p(\theta)-c] D_{\theta}$.

(ii) The regulated price, $p(\theta)$ is a nondecreasing function of $\theta$.

The proof of Lemma 1 is standard and is omitted. See, for example, Baron and Myerson (1982) and Guesnerie and Laffont (1984). The next Lemma constrains the set of optimal regulatory price policies.

LEMMA 2: The optimal regulatory prices under private information must cover marginal cost. That is, $p(\theta)-c \geq 0 \forall \theta \in[\underline{\theta}, \bar{\theta}]$.

Proof: Assume that $p(\theta)$ is a solution to the regulatory problem such that $\tilde{p}(\theta)<c$ for $\theta \in[\underline{\theta}, \tilde{\theta}] .{ }^{9}$ At $\theta \in[\underline{\theta}, \tilde{\theta}]$ the slope of $\Pi(\theta)$ required to

${ }^{9}$ Notice that $p(\tilde{\theta})<c$ implies $\left.\not \partial \theta\right)<c$ for $\theta \in[\underline{\theta}, \tilde{\theta}]$ given part (ii) in Lemma 1 . 
induce truthtelling, given part (i) in Lemma 1, is

$$
\frac{d \Pi(\theta)}{d \theta}=\Pi_{\theta}=[\tilde{p}(\theta)-c] D_{\theta}<0,
$$

which specifies how informational rents must change with the demand parameter. Individual rationality, $\Pi(\theta) \geq 0 \forall \theta \in \Theta$, and (P1) implies that any type $\theta \in[\underline{\theta}, \tilde{\theta}]$ would obtain a strictly positive information rent. Consider a price policy $\tilde{\tilde{p}}(\theta)$ such that

$$
\tilde{p}(\theta)= \begin{cases}\tilde{p}(\theta) & \text { for } \theta \in(\tilde{\theta}, \bar{\theta}] \\ c & \text { for } \theta \in[\underline{\theta}, \tilde{\theta}] .\end{cases}
$$

Note that by moving from $\tilde{p}(\theta)$ to $\tilde{\tilde{p}}(\theta)$ total surplus increases, since from a social welfare point of view prices must be greater than marginal cost because of the cost of public funds, and informational rents also decreases. Therefore, this argument contradicts $\tilde{p}(\theta)$ as a solution to the regulatory problem.

Lemma 1 characterizes the class of incentive compatible contracts. This lemma implies that any nondecreasing price function induces truthtelling if the corresponding transfer function is such that the firm's profit (information rents) varies with $\theta$ accordingly with condition (i). The informational rents are due solely to private information, and they result because a firm with a demand parameter $\theta$ has an incentive to report its type as $\hat{\theta} \neq \theta$ when prices differ from marginal cost. Condition (i) in Lemma 1 implies that the firm has an incentive to understate its demand parameter when its corresponding price level exceeds marginal cost and an incentive to overstate its demand parameter in the opposite case. Under private information on demand, the regulator does not choose the full information price (which is feasible given that $p^{*}(\theta)$ is an increasing function of $\theta$ ) because this price policy would generate excessive firm's informational rents. Since information rents depend on the difference between price and marginal cost, the regulator can reduce those rents by decreasing this difference. The gains from reducing information rents come at the expense of a reduction in total surplus. The optimal tradeoff between minimizing informational rents and maximizing total surplus sets a limit on the price reduction. A price reduction below marginal cost would generate more information rents and a higher loss in total surplus than a price policy that equates marginal cost.

Lemma 2 implies that $d \Pi(\theta) / d \theta \geq 0 \forall \theta$, so individual rationality constraints need be satisfied only at $\theta=\underline{\theta}$ and this therefore allows us to rewrite condition (i) of Lemma 1 as

$$
\Pi(\theta)=\Pi(\underline{\theta})+\int_{\underline{\theta}}^{\theta} \Pi_{\theta}(p(z), z) d z .
$$


The regulation problem can be thus rewritten as

$$
\begin{aligned}
\max _{p(\theta), \Pi(\theta)} \int_{\theta \in[\theta, \bar{\theta}]}\left\{\int_{p}^{\infty} D(x, \theta) d x+(1+\lambda)[p(\theta) D(p(\theta), \theta)\right. \\
-c D(p(\theta), \theta)-F]-\lambda \Pi(p(\theta), \theta)\} f(\theta) d \theta
\end{aligned}
$$

subject to $\quad \Pi(\theta)=\Pi(\underline{\theta})+\int_{\underline{\theta}}^{\theta} \Pi_{\theta}(p(z), z) d z$

$$
\begin{aligned}
\Pi(\underline{\theta}) & \geq 0 \\
\frac{d p(\theta)}{d \theta} & \geq 0 \\
p(\theta)-c & \geq 0 \quad \forall \theta \in[\underline{\theta}, \bar{\theta}] .
\end{aligned}
$$

By introducing (a) into the objective function and by taking into account that (b) is binding at the optimum, we can write the regulator's maximization problem as

$$
\begin{gathered}
\max _{p(\theta)} \int_{\underline{\theta}}^{\bar{\theta}}\left\{\int_{p}^{\infty} D(x, \theta) d x+(1+\lambda)[p D(p, \theta)-c(D(p, \theta))-F]\right. \\
\left.-\lambda \frac{1-F(\theta)}{f(\theta)} \Pi_{\theta}(p(\theta), \theta)\right\} f(\theta) d \theta .
\end{gathered}
$$

Firstly, we solve the problem without taking into account constraints (c) and (d) and then we analyze the implications of these restrictions on the optimal regulatory policy. The first order condition is given by

$$
\begin{aligned}
\frac{d S W R}{d p}= & \left.\left.\{-D(\hat{p}(\theta), \theta))+[D(\hat{p}(\theta), \theta))+(\hat{p}(\theta)-c) D_{p}(\hat{p}(\theta), \theta)\right]\right\} f(\theta) \\
& \left.+\lambda[D(\hat{p}(\theta), \theta))+(\hat{p}(\theta)-c) D_{p}(\hat{p}(\theta), \theta)\right] f(\theta) \\
& \left.-\lambda[1-F(\theta)] \Pi_{\theta p}(\hat{p}(\theta), \theta)\right) \\
= & 0
\end{aligned}
$$

With respect to the full information case, under private information on demand there is an additional effect: the fourth term in (12). A marginal rise in type $\theta$ 's price (the firm's price with demand parameter $\theta$ ) generates a uniform increase in transfer to any firm $\theta^{\prime}>\theta$ that is needed to restore incentive compatibility. The social cost of these transfers in the upper tail of the distribution is $\lambda[1-F(\theta)] \Pi_{\theta p}(\cdot)$. This additional cost implies that for any demand parameter $\theta \in[\underline{\theta}, \bar{\theta})$ the price chosen by the regulator is smaller than the price under full information. As we have pointed out, marginal cost constitutes a lower bound to the regulatory price under private information, 
that is, condition (d) must be satisfied. It is easy to check that (c) is satisfied under the monotone hazard rate condition. We bring together our results in the following proposition.

PROPOSITION 1: Theoptimal regulatory price policy underincomplete information, $p^{p i}(\theta)$, is given by

$$
p^{p i}(\theta)= \begin{cases}\hat{p}(\theta) & \text { for } \bar{\theta} \geq \theta \geq \theta_{o} \\ c & \text { for } \theta_{o} \geq \theta\end{cases}
$$

where $\hat{p}(\theta)$ solves

$$
\frac{\hat{p}(\theta)-c}{\hat{p}(\theta)}=\frac{\lambda}{1+\lambda}\left[\frac{1}{\varepsilon}+\frac{1-F(\theta)}{f(\theta)} \frac{\Pi_{\theta p}}{p D_{p}}\right]
$$

and $\theta_{o}$ is such that

$$
D\left(\hat{p}\left(\theta_{o}\right), \theta_{o}\right)-\frac{1-F\left(\theta_{o}\right)}{f\left(\theta_{o}\right)} \Pi_{\theta p}\left(\hat{p}\left(\theta_{o}\right), \theta_{o}\right)=0 .
$$

Proof: Firstly, if we do not take into account constraint (d) then it is straightforward to obtain the price policy $\hat{p}(\theta)$ in (13) from condition (12). If we include constraint $(d)$ then the optimal regulatory policy may be expressed as $p^{p i}(\theta)=\max \{\hat{p}(\theta), c\}$. Denote as $\theta_{o}$ the type such that condition (12) is satisfied but the price is equal to marginal cost, that is, $\hat{p}\left(\theta_{o}\right)=c$ and $f\left(\theta_{o}\right) D\left(\hat{p}\left(\theta_{o}\right), \theta_{o}\right)=\left[1-F\left(\theta_{o}\right)\right] \Pi_{\theta p}\left(\hat{p}\left(\theta_{o}\right), \theta_{o}\right)$. By evaluating marginal social welfare at a price $p(\theta)=c$ we obtain

$$
\begin{aligned}
\frac{d \operatorname{SWR}(p(\theta)=c)}{d p}= & f(\theta) D(p(\theta), \theta) \\
& -(1-F(\theta)) \Pi_{\theta p}(p(\theta), \theta)>0 \quad \forall \theta>\theta_{o} \\
\frac{d \operatorname{SWR}(p(\theta)=c)}{d p}= & f(\theta) D(p(\theta), \theta) \\
& -(1-F(\theta)) \Pi_{\theta p}(p(\theta), \theta)<0 \quad \forall \theta<\theta_{o}
\end{aligned}
$$

because $d \operatorname{SWR}(p(\underline{\theta})=c) / d p$ is an increasing function of $\theta$. This implies that for any $\theta>\theta_{o}$ there is a social benefit (a saving of public funds) from increasing price beyond marginal cost which implies $p^{p i}(\theta)=\hat{p}(\theta)$ for $\bar{\theta} \geq \theta \geq \theta_{o}$. However, for types $\theta<\theta_{o}$ an increase in price beyond marginal cost generates a loss of social welfare and, given Lemma 2 , the price must be equal to marginal cost, $p^{p i}(\theta)=c$ for $\theta_{o} \geq \theta$.

It must be stressed that $\bar{\theta}>\theta_{o}$ (so at least for types at the upper end of the support price will always be over marginal cost) and that $\theta_{o}$ may be lower than $\underline{\theta}\left(p^{p i}(\theta)=\hat{p}(\theta)>c\right.$ for any $\theta \in[\underline{\theta}, \bar{\theta}]$ when $\left.d \operatorname{SWR}(p(\underline{\theta})=c) / d p>0\right)$. 
Note that the critical type $\theta_{o}$ crucially depends on the ratio $f(\theta) /[1-F(\theta)]$ but is, however, independent of the cost of public funds. When the regulator chooses the optimal price under private information on demand, the benchmark is $p^{*}(\theta)$ (the full information price) which is such that $p^{*}(\theta)>c$ because of the cost of public funds. Private information generates information rents that can be reduced by decreasing the difference between price and marginal cost, but this reduction implies a loss in total surplus. As we have pointed out above, the regulator faces a tradeoff between maximizing total surplus and appropriating the firm's informational rents. Values of the demand parameter close to $\bar{\theta}$ have high ratios $f(\theta) /[1-F(\theta)]$. In these cases, by reducing price over the interval $[\theta, \theta+d \theta]$ the total surplus (which has a weight $f(\theta)$ ) is substantially reduced in expected value compared with the expected decrease in information rents (with weight $1-F(\theta)$ ) of types in $[\theta+d \theta, \bar{\theta}]$. The optimal tradeoff implies small deviation in prices on the upper side of the support. When $\theta$ decreases, the monotone hazard rate property implies that $f(\theta) /[1-F(\theta)]$ also decreases and, therefore, the optimal price reduction (with respect to the full information case) is higher. When $f(\theta) /[1-$ $F(\theta)]$ is small enough, which occurs for types $\theta \leq \theta_{0}$, price equals marginal cost.

The following corollary states some properties of the optimal regulatory policy.

COROLLARY 1: The optimal regulatory policy under incomplete information entails

(a) an efficient price level for type $\bar{\theta}$ (nondistortion at the top);

(b) a price lower than the efficient one for any type $\theta \in[\underline{\theta}, \bar{\theta})$;

(c) a decrease of the Lerner index for any type $\theta \in[\underline{\theta}, \bar{\theta})$; and

(d) an informational rent for any type $\theta \in\left(\theta_{o}, \bar{\theta}\right]$.

Proof: The marginal expected welfare (under incomplete information) at the optimal price under complete information, $p^{*}(\theta)$, is negative for any type $\theta \in[\underline{\theta}, \bar{\theta})$. Given the concavity of the objective function we have $p^{p i}(\theta)<p^{*}(\theta)$ for any $\theta \in[\underline{\theta}, \bar{\theta})$. Moreover, the first order conditions under complete and incomplete information coincide when $\theta=\bar{\theta}$, which implies $p^{p i}(\bar{\theta})=p^{*}(\bar{\theta})$. On the other hand, given the single-crossing property (which implies $\Pi_{\theta p}>0$ ) we obtain from (13) that the Lerner index decreases (with respect to the full information case). Given that social welfare decreases with firms' profits we obtain from (a), (b) and (d) that $\Pi(\theta)=0$ for any $\theta \in\left[\underline{\theta}, \theta_{o}\right]$ and $\Pi(\theta)>0$ for any $\theta \in\left(\theta_{o}, \bar{\theta}\right]$, which completes the proof.

The possibility of type $\theta \in\left(\theta_{o}, \bar{\theta}\right]$ mimicking the behavior of the lower demand types leads the regulator to give up a positive rent to the first one. If the regulator were to insist on the optimal prices under full information, the result would be excessively high informational rents. In order to reduce these rents, prices are downward distorted from the optimal prices under full information, that is, $p^{p i}(\theta)<p^{*}(\theta)$ for any $\theta \in[\underline{\theta}, \bar{\theta})$. 


\section{A Generalized Social Welfare Function}

In this section, we consider a social welfare function that incorporates both distributional considerations and costly public funds

$$
S W_{G}(p, t)=C S(p, t)+\alpha \Pi(p, t),
$$

where $\alpha \in[0,1]$ and the net surplus of consumers and the profit of the firm are given respectively by (2) and (3).

Under full information results are the same as those in Section 3, that is, the optimal regulatory price is given by the Ramsey formula (10). Under private information on demand, Lemmas 1 and 2 apply and following the lines in Section 4 we can rewrite the regulation problem as

$$
\begin{gathered}
\max _{p(\theta)} \int_{\underline{\theta}}^{\bar{\theta}}\left\{\int_{p}^{\infty} D(x, \theta) d x+(1+\lambda)[p D(p, \theta)-c(D(p, \theta))-F]\right. \\
\left.-(1-\alpha+\lambda) \frac{1-F(\theta)}{f(\theta)} \Pi_{\theta}(p(\theta), \theta)\right\} f(\theta) d \theta
\end{gathered}
$$

subject to (c) and (d).

As in the previous section we characterize the optimal regulatory price policy under private information on demand in the following proposition.

PROPOSITION 2: The optimal regulatory price policy under private information on demand, $p_{G}^{p i}(\theta)$, is given by

$$
p_{G}^{p i}(\theta)=\left\{\begin{array}{lll}
\hat{p}_{G}(\theta) & \text { for } & \bar{\theta} \geq \theta \geq \theta_{o}^{G} \\
c & \text { for } & \theta_{o}^{G} \geq \theta
\end{array}\right.
$$

where $\hat{p}_{G}(\theta)$ solves

$$
\begin{aligned}
\frac{\hat{p}_{G}(\theta)-c}{\hat{p}_{G}(\theta)}= & \frac{\lambda}{1+\lambda}\left[\frac{1}{\varepsilon}+\frac{1-F(\theta)}{f(\theta)} \frac{\Pi_{\theta p}}{\hat{p}_{G}(\theta) D_{p}}\right] \\
& +\frac{(1-\alpha)}{1+\lambda} \frac{1-F(\theta)}{f(\theta)} \frac{\Pi_{\theta p}}{\hat{p}_{G}(\theta) D_{p}}
\end{aligned}
$$

and $\theta_{o}^{G}$ is such that

$$
\begin{gathered}
\lambda\left[D\left(c, \theta_{o}^{G}\right)-\frac{1-F\left(\theta_{o}^{G}\right)}{f\left(\theta_{o}^{G}\right)} \Pi_{\theta p}\left(c, \theta_{o}^{G}\right)\right] \\
-(1-\alpha) \frac{1-F\left(\theta_{o}^{G}\right)}{f\left(\theta_{o}^{G}\right)} \Pi_{\theta p}\left(c, \theta_{o}^{G}\right)=0 .
\end{gathered}
$$

Proof: The proof is almost identical to the proof of Proposition 1.

According to Proposition 2 we can distinguish the following cases depending on $\lambda$ and $\alpha$ : 
(i) If $\lambda=0$ and $\alpha=1$ then $p_{G}^{p i}(\theta)=c \forall \theta \in \Theta$. That is, private information has no effects when the standard first-best analysis is considered.

(ii) If $\lambda=0$ and $\alpha<1$ then $p_{G}^{p i}(\theta)=c \forall \theta \in \Theta$, and $\theta_{o}^{G}=\bar{\theta}$. This is the case considered by Lewis and Sappington (1988a). If there is no cost of public funds the full information policy (which coincides with the first best policy) is implementable and private information is inconsequential for regulation.

(iii) If $\lambda>0$ and $\alpha=1$ then $p_{G}^{p i}(\theta)=p^{p i}(\theta)$ and $\theta_{o}^{G}=\theta_{o}$. This case is considered in Section 4.

(iv) If $\lambda>0$ and $\alpha \in[0,1)$ then $p_{G}^{p i}(\theta) \leq p^{p i}(\theta) \forall \theta \in \Theta$ and $\theta_{o}^{G}(\lambda, \alpha) \geq$ $\theta_{o}$. It is easy to check from condition (17) that $\frac{\partial \theta_{o}^{G}(\lambda, \alpha)}{\partial \lambda}<0$ and $\frac{\partial \theta_{o}^{G}(\lambda, \alpha)}{\partial \alpha}<0$. When $\lambda>0$, a decrease in $\alpha$ implies a smaller weight of the firm's profit in social welfare. Informational rents become more costly and therefore the regulator finds it optimal to reduce the difference between price and marginal cost. This implies that the set of types for which price equals marginal cost becomes larger. However, even though $\alpha=0$ there will always be a nonnegligible set of types for which price exceeds marginal cost.

Note that under costly public funds (cases (iii) and (iv)) the full information price policy is given by the Ramsey formula and therefore the price exceeds marginal cost. If the regulator is less well informed about the market demand than the firm, the presence of informational rents generates additional social costs that prevent the Ramsey pricing rule from being established. To reduce informational rents it is optimal to reduce the difference between price and marginal cost and, moreover, the optimal price under private information may be equal to marginal cost for types $\theta \in\left[\underline{\theta}, \theta_{0}^{G}\right]$. But even for these types our results and those of Lewis and Sappington (1988a) are qualitatively different. Under costly public funds the full information benchmark differs from the private information price rule, which implies that there is a social welfare loss due to the asymmetry of information. Under distributional considerations (Lewis and Sappington 1988a) with no costs of public funds, marginal cost pricing characterizes both the full information case and the private information setting, which implies that private information has no social value.

\section{Concluding Remarks}

In this paper, we have analyzed the optimal regulation policy when the regulated firm has better information concerning the market demand than the regulator. We show that introducing a cost of public funds into the Planner's objective function does not lead to qualitative results similar to those obtained by introducing distributional considerations. In particular, we show that 
under constant marginal cost the full information policy is not implementable and that the optimal regulatory policy implies price distortions in order to reduce informational rents.

Contrary to Lewis and Sappington (1988a), under private information concerning demand, the presence of costly public funds leads to similar qualitative results to those obtained under private cost information in the sense that asymmetric information precludes the achievement of optimal pricing rule. This means that under private information about demand it is not possible to simply transfer the conclusions from a model where distributional concerns are taken into account to one with a shadow price of public funds.

Our model could be generalized in several directions. The assumption of constant marginal cost could be relaxed in order to allow (strictly) increasing marginal cost without altering the main results of the paper. On the other hand, a possible extension of the paper that we leave for further research would be to consider the optimal regulation of a firm with private information about its cost and demand conditions under costly public funds.

\section{References}

ARMSTRONG, M. (1999) Optimal regulation with unknown demand and cost functions, Journal of Economic Theory 84, 196-215.

ATKINSON, A., and J. STIGLITZ (1980) Lectures on Public Economics. New York: McGraw-Hill.

BALLARD, C. L., J. B. SHOVEN, and J. WHALLEY (1985) General equilibrium computations of the marginal welfare costs of taxes in the United States, American Economic Review 75, 128-138.

BARON, D. (1989) Design of Regulatory Mechanisms and Institutions, in Handbook of Industrial Organization, Vol. II, R. Schmalensee and R. Willig, eds. Amsterdam: North-Holland.

BARON, D., and R. MYERSON (1982) Regulating a monopolist with unknown costs, Econometrica 50, 911-930.

BIGLAISER, G., and C. A. MA (1995) Regulating a dominant firm: Unknown demand and industry structure, Rand Journal of Economics 26, 1-19.

CAILLAUD, B., R. GUESNERIE, P. REY, and J. TIROLE (1988) Goverment intervention in production and incentives theory: A review of recent contributions, Rand Journal of Economics 19, 1-26.

DASGUPTA, P., P. HAMMOND, and E. MASKIN (1979) The implementation of social choice rules: Some general results on incentive compatibility, Review of Economic Studies 46, 185-216.

GUESNERIE, P., and J.-J. LAFFONT (1984) A complete solution to a class of principalagent problems with an application to the control of a self-managed firm, Journal of Public Economics 25, 329-369. 
LAFFONT, J.-J., and J. TIROLE (1986) Using cost observation to regulate firms, Journal of Political Economy 94, 614-641.

LAFFONT, J.J., and J. TIROLE (1993) A Theory of Incentives in Procurement and Regulation. Cambridge, MA: MIT Press.

LEWIS, T. R., and D. E. M. SAPPINGTON (1988a) Regulating a monopolist with unknown demand, American Economic Review 78, 986-998.

LEWIS, T. R., and D. E. M. SAPPINGTON (1988b) Regulating a monopolist with unknown demand and cost functions, Rand Journal of Economics 19, 438-457.

MYERSON, R. (1979) Incentive-compatibility and the bargaining problem, Econometrica 47, 61-74.

RIORDAN, M. (1984) On delegating price authority to a regulated firm, Rand Journal of Economics 15, 108-115. 